\title{
A Definition of Lattice Implication Algebra Based on Implication Operator
}

\author{
Liangzhong Yi Zheng Pei \\ School of Mathematics \& Computer Science, Xihua University, Chengdu 610039, P.R. China
}

\begin{abstract}
Lattice implication algebra is an important nonclassical logical algebra, it has been studied by researchers. Binary operation $\wedge, \vee$ and unitary operation ' in lattice implication algebra could be defined by implication operation $\rightarrow$, namely, these operations in lattice implication algebra are not independently. In this paper, firstly, we use implication operation $\rightarrow$ to define binary operation $\wedge, \vee$ and unitary operation ', then, partial set and lattice could be constructed, finally, another definition of lattice implication algebra is discussed.
\end{abstract}

Keywords: Lattice implication algebra, Binary operation, Partial set

\section{Introduction}

To establish an alternative logic for knowledge representation and reasoning, $\mathrm{Xu}$ [1] proposed a logical algebra-lattice implication algebra in 1993 by combining algebraic lattice and implication algebra. In lattice implication algebra, the lattice is defined to describe uncertainties, especially for the incomparability, and the implication operator is designed to describe the way of human's reasoning. $\mathrm{Xu}$ et al., have established the lattice-valued propositional logic $L P(X)$ [2], [3] and lattice-valued firstorder logic $L F(X)$ [4], the gradual lattice-valued propositional logic $L v p l$ and the gradual latticevalued first-order logic $L v f l$ [5]-[7] by taking lattice implication algebra as truth-value field. Lattice implication algebra, lattice-valued logic based on lattice implication algebra are collected in [8]. Formally, lattice implication algebra could be defined as following

Definition 1 Let $(L, \vee, \wedge, O, I)$ be a bounded lattice with order-reversing involution ', $I$ and $O$ be the greatest and the least elements of $L$, respectively, $\rightarrow: L \times L \longrightarrow L$ be a mapping and satisfy the following conditions: for any $x, y, z \in L$ :

$$
\begin{aligned}
& I_{1} \quad: \quad x \rightarrow(y \rightarrow z)=y \rightarrow(x \rightarrow z) \\
& I_{2} \quad: \quad x \rightarrow x=I \\
& I_{3} \quad: \quad x \rightarrow y=y^{\prime} \rightarrow x^{\prime} \\
& I_{4} \quad: \quad x \rightarrow y=y \rightarrow x \quad \text { implies } \quad x=y \\
& I_{5} \quad: \quad(x \rightarrow y) \rightarrow y=(y \rightarrow x) \rightarrow x \\
& I_{6} \quad: \quad(x \vee y) \rightarrow z=(x \rightarrow z) \wedge(y \rightarrow z) \\
& I_{7} \quad: \quad(x \wedge y) \rightarrow z=(x \rightarrow z) \vee(y \rightarrow z)
\end{aligned}
$$

Then $\left(L, \vee, \wedge,{ }^{\prime}, \rightarrow, O, I\right)$ is called a lattice implication algebra.

In this paper, based on binary operation $\rightarrow$, the greatest element and the least element, an equivalent definition of lattice implication algebra is discussed.

\section{Generating lattice based on implication operator on $L$}

Let $L$ be a non-empty set, and $I \in L$ be a fixed element, $\rightarrow$ be a binary operation in $L . \forall x, y \in L$, let

$$
x * y=(x \rightarrow y) \rightarrow y
$$

$*$ is a binary operation in $L$ derived from (1) according to $\rightarrow$ in $L$.

Lemma 1 Let $L$ be a non-empty set, $I \in L$ be a fixed element, and $\rightarrow$ be a binary operation in $L$. $\forall x, y, z \in L$, if $\rightarrow$ satisfies the following conditions

$$
\begin{array}{ll}
\left(L_{1}\right) & I \rightarrow x=x \\
\left(L_{2}\right) & x \rightarrow x=I \\
\left(L_{3}\right) & (x \rightarrow y) \rightarrow y=(y \rightarrow x) \rightarrow x \\
\left.\left(L_{4}\right) \quad((y \rightarrow z) \rightarrow z) \rightarrow x\right) \rightarrow x \\
\quad \quad \quad(((y \rightarrow x) \rightarrow x) \rightarrow z) \rightarrow z
\end{array}
$$

then $(L, *)$ is a half-lattice and $x * y=\sup \{x, y\}=$ $x \vee y$. 
Proof 1) $\forall x \in L$, according to $\left(L_{1}\right)$ and $\left(L_{2}\right)$, idempotent law come into existence, namely $x * x=(x \rightarrow$ $x) \rightarrow x=I \rightarrow x=x$.

2) $\forall x, y \in L$, according to $\left(L_{3}\right)$, exchange law come into existence, namely $x * y=(x \rightarrow y) \rightarrow y=$ $(y \rightarrow x) \rightarrow x=y * x$.

3) $\forall x, y, z \in L$, according to $\left(L_{3}\right)$ and $\left(L_{4}\right)$, coalescence law come into existence, namely

$$
\begin{aligned}
x *(y * z) & =(y * z) * x \\
& =(((y \rightarrow z) \rightarrow z) \rightarrow x) \rightarrow x \\
& =(((y \rightarrow x) \rightarrow x) \rightarrow z) \rightarrow z \\
& =((y * x) \rightarrow z) \rightarrow z \\
& =((x * y) \rightarrow z) \rightarrow z \\
& =(x * y) * z .
\end{aligned}
$$

According to $(1),(2)$ and $(3),(L, *)$ is a half-lattice. On the other hand, $\leq$ can be defined as follows: $\forall x, y \in L$,

$\left(F_{1}\right) \quad x \leq y \quad$ iff $\quad x * y=(x \rightarrow y) \rightarrow y=y$

$\leq$ is a partial relation which can be proved by the following, $\forall x, y, z \in L$,

4) According to $x * x=x, x \leq x$ can be obtained, so reflexivity come into existence;

5) If $x \leq y$ and $y \leq x$, according to $\left(F_{1}\right)$ and $*$ satisfying exchange law, then dissymmetry come into existence, namely $y=x * y=y * x=x$.

6) If $x \leq y$ and $y \leq z$, according to formula (??) and $*$ satisfying coalescence law, then transfer law come into existence, namely $x * z=x *(y * z)=$ $(x * y) * z=y * z=z$.

so, $x \leq z$. According to $(4),(5)$ and $(6),(L, \leq)$ is a partial set. Also $\forall x, y \in L, x *(x * y)=(x * x) *$ $y=x * y$, namely $x \leq x * y, y *(x * y)=(y * x) * y=$ $(x * y) * y=x *(y * y)=x * y$, namely $y \leq x * y$.

Supposed that $\exists z \in L$ satisfy $x \leq z$ and $y \leq z$, then $(x * y) * z=x *(y * z)=x * z=z$, namely $x * y \leq z$, so, $x * y$ is the upper boundary of $x$ and $y$, namely $x * y=\sup \{x, y\}=x \vee y$.

As the above description, it can be proved that $(L, *)$ is a half-lattice, and $\forall x, y \in L, x * y=$ $\sup \{x, y\}=x \vee y$.

Theorem 1 Let $L$ be a non-empty set, $I \in L$ be a fixed element, $\rightarrow$ be a binary operation in $L$. $\forall x, y, z \in L, \rightarrow$ satisfy $\left(L_{1}\right)-\left(L_{4}\right)$, then

(1) $\forall x \in L, x \leq I$;

(2) If $x \rightarrow y=y \rightarrow x=I$, then $x=y$.

Proof 1) $\forall x \in L$, according to $\left(L_{1}\right)$ and $\left(L_{2}\right)$, it can be obtained $x \vee I=(x \rightarrow I) \rightarrow I=(I \rightarrow x) \rightarrow$ $x=x \rightarrow x=I$. According to formula (2), $x \leq I$ can be obtained.

2) If $x \rightarrow y=y \rightarrow x=I$, according to $\left(L_{1}\right)$, it can be obtained $y=I \rightarrow y=(x \rightarrow y) \rightarrow y=$ $y \rightarrow x \rightarrow x=I \rightarrow x=x$.

Theorem 2 Let $L$ be a non-empty set, $I \in L$ be a fixed element, $\rightarrow$ be a binary operation in $L$. $\forall x, y, z \in L, \rightarrow$ satisfy $\left(L_{1}\right)-\left(L_{4}\right)$ and

$$
\left(L_{5}\right) x \rightarrow(y \rightarrow z)=y \rightarrow(x \rightarrow z),
$$

then $x \leq y$ iff $x \rightarrow y=I$.

Proof 1) If $x \leq y$, then $x \vee y=y$. According to $\left(L_{2}\right)$ and $\left(L_{5}\right)$, it can be obtained

$$
\begin{aligned}
x \rightarrow y & =x \rightarrow(x \vee y)=x \rightarrow((x \rightarrow y) \rightarrow y) \\
& =(x \rightarrow y) \rightarrow(x \rightarrow y)=I
\end{aligned}
$$

2) If $x \rightarrow y=I$, then according to $\left(L_{1}\right)$, it can be obtained $x \vee y=(x \rightarrow y) \rightarrow y=I \rightarrow y=y$, so $x \leq y$.

According to the conclusion of Theorem 1 and Theorem 2, it can be obtained the following.

Corollary 1 Let $L$ be a non-empty set, $I \in L$ be a fixed element, $\rightarrow$ be a binary operation in $L$. $\forall x, y, z \in L, \rightarrow$ satisfy $\left(L_{1}\right)-\left(L_{5}\right)$, then $\forall x \in L$, $x \rightarrow I=I$.

Theorem 3 Let $L$ be a non-empty set, $I \in L$ be a fixed element, $\rightarrow$ be a binary operation in $L$ and satisfy $\left(L_{1}\right)-\left(L_{5}\right)$, then we have the following conclusions,

(1) If $x, y \in L$ and $x \leq y$, then $\forall z \in L, y \rightarrow z \leq$ $x \rightarrow z$, namely $(y \rightarrow z) \rightarrow(x \rightarrow z)=I$.

(2) $\forall x, y \in L$, if $x \leq y$, then $\forall z \in L, z \rightarrow x \leq z \rightarrow$ $y$, namely $(z \rightarrow x) \rightarrow(z \rightarrow y) \leq I$.

(3) If $x, y \in L$ and $x \leq y$, then $\forall z \in L, x \leq z \rightarrow y$, namely $x \rightarrow(z \rightarrow y)=I$.

Proof 1) According to $x \leq y$, it can be obtained $x \rightarrow y=I$. For $\forall z \in L$, according to formula (??), $\left(L_{3}\right)$ and $\left(L_{5}\right)$, it can be obtained

$$
\begin{aligned}
x \rightarrow(z \vee y) & =x \rightarrow((y \rightarrow z) \rightarrow z) \\
& =(y \rightarrow z) \rightarrow(x \rightarrow z) \\
& =x \rightarrow((z \rightarrow y) \rightarrow y) \\
& =(z \rightarrow y) \rightarrow(x \rightarrow y) \\
& =(z \rightarrow y) \rightarrow I=I,
\end{aligned}
$$

hence, $y \rightarrow z \leq x \rightarrow z$. 
2) For $x \leq y$, then $\forall z \in L$,

$$
\begin{aligned}
z \rightarrow y & =z \rightarrow(x \vee y) \\
& =z \rightarrow((y \rightarrow x) \rightarrow x) \\
& =(y \rightarrow x) \rightarrow(z \rightarrow x) \geq z \rightarrow x .
\end{aligned}
$$

3) For $x \leq y$, according to Theorem 3 (2), it can be obtained $z \rightarrow y \geq z \rightarrow x$. According to Theorem 3 (1), it can be obtained $z \rightarrow x \geq I \rightarrow x=x$, so $x \leq z \rightarrow y$.

Theorem 4 Let $L$ be a non-empty set, $I \in L$ be a fixed element, $\rightarrow$ be a binary operator in $L$ and satisfy $\left(L_{1}\right)-\left(L_{5}\right)$. If there exists an element $O$ in $L$, and satisfy $\forall x \in L, O \leq x$, then it can be obtained,

(1) $\forall x \in L,(x \rightarrow O) \rightarrow O=x$;

(2) $\forall x, y \in L, x \rightarrow y=(y \rightarrow O) \rightarrow(x \rightarrow O)$.

Proof 1) According to $O \leq x$, it can be obtained $x=I \rightarrow x=(O \rightarrow x) \rightarrow x=(x \rightarrow O) \rightarrow O$.

2) According to $\left(L_{5}\right)$, it can be obtained $(y \rightarrow$ $O) \rightarrow(x \rightarrow O)=x \rightarrow((y \rightarrow O) \rightarrow O)=x \rightarrow$ $(y \vee O)=x \rightarrow y$.

According to the element $O$ and $\rightarrow$ in $L$, we can define the following mapping from $L$ to $L$ :

$$
\text { ' }: L \rightarrow L, x \mapsto x^{\prime}=x \rightarrow O
$$

Theorem 5 The mapping' decided by $\left(F_{2}\right)$ from $L$ to $L$ is reversal involution mapping.

Proof $\forall x, y \in L$, if $x \leq y$, according to the conclusion (1) of 3 , it can be obtained $y^{\prime}=y \rightarrow O \leq x \rightarrow$ $O=x^{\prime}$. Hence, ${ }^{\prime}$ is reversal.

On the other hand, if $x \rightarrow O \neq y \rightarrow O$, then $x \neq y$. Else if $x=y$, then $(x \rightarrow O) \rightarrow(y \rightarrow$ $O)=y \rightarrow x=I=x \rightarrow y=(y \rightarrow O) \rightarrow(x \rightarrow O)$. But it is not consistent with $x \rightarrow O=y \rightarrow O$. So ' ${ }^{\prime}$ is a one to one mapping. According to the conclusion (1) of Theorem 4, it can be obtained $\left(x^{\prime}\right)^{\prime}=(x \rightarrow O) \rightarrow O=x$. So, ${ }^{\prime}$ is a involution in $L$. According to the above description, ${ }^{\prime}$ is reversal involution mapping in $L$.

In the following discussion in $L, x \rightarrow O$ are all represented by $x^{\prime}$. Especially, according to $\left(L_{1}\right)$ and $\left(L_{2}\right)$, it can be obtained $I^{\prime}=I \rightarrow O=O$, $O^{\prime}=O \rightarrow O=I$. And (2) in Theorem 5 can be rewritten by

$\left(F_{3}\right) \quad \forall x, y \in L, x \rightarrow y=y^{\prime} \rightarrow x^{\prime}$.

If $L$ satisfy the conditions in Theorem 4, then import the following mark in $L x \wedge y=\left(x^{\prime} \vee y^{\prime}\right)^{\prime}$. The following conclusion can be proved.
Theorem 6 Let $L$ be a non-empty set, $I \in L$ be a fixed element, $\rightarrow$ be a binary operator in $L$ and satisfy $\left(L_{1}\right)-\left(L_{5}\right)$. If there exists an element $O$ in $L$, and satisfy $\forall x \in L, O \leq x$, then it can be obtained

$$
x \wedge y=\left(x^{\prime} \vee y^{\prime}\right)^{\prime}=\inf \{x, y\} .
$$

Proof According to $\left(F_{3}\right)$ and Theorem 2, it can be obtained

$$
\begin{gathered}
\left(x^{\prime} \vee y^{\prime}\right)^{\prime} \rightarrow x=x^{\prime} \rightarrow\left(x^{\prime} \vee y^{\prime}\right)=I, \\
\left(x^{\prime} \vee y^{\prime}\right)^{\prime} \rightarrow y=y^{\prime} \rightarrow\left(x^{\prime} \vee y^{\prime}\right)=I .
\end{gathered}
$$

Hence, $\left(x^{\prime} \vee y^{\prime}\right)^{\prime} \leq x$ and $\left(x^{\prime} \vee y^{\prime}\right)^{\prime} \leq y$. Supposed that there exists $l \in L$ satisfying $l \leq x$ and $l \leq y$, then $l^{\prime} \geq x^{\prime}$ and $l^{\prime} \geq y^{\prime}, l^{\prime} \geq x^{\prime} \vee y^{\prime}$. So $l \leq$ $\left(x^{\prime} \vee y^{\prime}\right)^{\prime}=x \wedge y$, namely

$$
x \wedge y=\left(x^{\prime} \vee y^{\prime}\right)^{\prime}=\inf \{x, y\} .
$$

Under the condition of Theorem 6 , it is easy to prove $(x \vee y)^{\prime}=x^{\prime} \wedge y^{\prime}$.

Corollary 2 Let $L$ be a non-empty set, $I \in L$ be a fixed element, $\rightarrow$ be a binary operator in $L$ and satisfy $\left(L_{1}\right)-\left(L_{5}\right)$. If there exists an element $O$ in L, and satisfy $\forall x \in L, O \leq x$, then it can be obtained

(1) $x \wedge x=x$;

(2) $x \wedge y=y \wedge x$;

(3) $(x \wedge y) \wedge z=x \wedge(y \wedge z)$.

Proof Here, we just prove (3).

$$
\begin{aligned}
(x \wedge y) \wedge z & =\left((x \wedge y)^{\prime} \vee z^{\prime}\right)^{\prime}=\left(\left(\left(x^{\prime} \vee y^{\prime}\right)^{\prime}\right)^{\prime} \vee z^{\prime}\right)^{\prime} \\
& =\left(\left(x^{\prime} \vee y^{\prime}\right) \vee z^{\prime}\right)^{\prime}=\left(x^{\prime} \vee\left(y^{\prime} \vee z^{\prime}\right)\right)^{\prime} \\
& =\left(x^{\prime} \vee\left(\left(y^{\prime} \vee z^{\prime}\right)^{\prime}\right)^{\prime}\right)^{\prime}=x \wedge\left(y^{\prime} \vee z^{\prime}\right)^{\prime} \\
& =x \wedge(y \wedge z) .
\end{aligned}
$$

Theorem 7 Let $L$ be a non-empty set, $I \in L$ be a fixed element, $\rightarrow$ be a binary operator in $L$ and satisfy $\left(L_{1}\right)-\left(L_{5}\right)$. If there exists an element $O$ in $L$, and satisfy $\forall x \in L, O \leq x$, then $(L, \vee, \wedge)$ is a boundary lattice.

Proof According to the conclusion (1) of Theorem 1 , it can be obtained that $I$ is the upper-boundary of $L$, and $O$ is its down-boundary. According to Lemma 1 and Corollary 2, it can be obtained that binary operation $\vee$ and $\wedge$ satisfy $x \wedge x=x, x \wedge y=$ $y \wedge x$ and $(x \wedge y) \wedge z=x \wedge(y \wedge z)$. In addition, according to $x \leq x \vee y$, it can be obtained $x \wedge(x \vee$ $y)=x$ and according to $x \geq x \wedge y$, then it can be 
obtained $x \vee(x \wedge y)=x$. So it satisfies absorption law, namely

$$
x \wedge(x \vee y)=x \vee(x \wedge y)=x
$$

According to the definition of boundary lattice, it can be obtained that $(L, \vee, \wedge)$ is a boundary lattice.

\section{An equivalent definition of lattice implication algebra}

In this Section, generating lattice implication algebra will be discussed on $(L, \vee, \wedge)$ based $\rightarrow$.

Corollary 3 Let $L$ be a non-empty set, $I \in L$ be a fixed element, $\rightarrow$ be a binary operator in $L$ and satisfy $\left(L_{1}\right)-\left(L_{5}\right)$. If there exists an element $O$ in $L$, and satisfy $\forall x \in L, O \leq x$, then for $x, y \in L$ and $x \leq y$, it can be obtained $\forall z \in L, z \rightarrow x \leq z \rightarrow y$.

Proof According to $x \leq y$, it can be obtained $x^{\prime} \geq y^{\prime} . \forall z \in L$, according to the conclusion (1) of Theorem 3, it can be obtained

$$
z \rightarrow x=x^{\prime} \rightarrow z^{\prime} \leq y^{\prime} \rightarrow z^{\prime}=z \rightarrow y
$$

Note 1 Under the condition, the Corollary can be proved by Theorem 3.

Theorem 8 Let $L$ be a non-empty set, $I \in L$ be a fixed element, $\rightarrow$ be a binary operator in $L$ and satisfy $\left(L_{1}\right)-\left(L_{5}\right)$. If there exists an element $O$ in $L$, and satisfy $\forall x \in L, O \leq x$, then $\forall x, y, z \in L$,

$$
(x \vee y) \rightarrow z=(x \rightarrow z) \wedge(y \rightarrow z)
$$

Proof 1) According to equation $1,\left(L_{5}\right)$ and Theorem 2 , it can be obtained

$$
\begin{array}{ll} 
& ((x \vee y) \rightarrow z) \rightarrow(x \rightarrow z) \\
= & x \rightarrow(((x \vee y) \rightarrow z) \rightarrow z) \\
=\quad & x \rightarrow(x \vee y \vee z)=I \\
& ((x \vee y) \rightarrow z) \rightarrow(y \rightarrow z) \\
=\quad & y \rightarrow(((x \vee y) \rightarrow z) \rightarrow z) \\
=\quad & y \rightarrow(x \vee y \vee z)=I .
\end{array}
$$

Hence, $(x \vee y) \rightarrow z \leq(x \rightarrow z) \wedge(y \rightarrow z)$.
2) $((x \rightarrow z) \wedge(y \rightarrow z)) \rightarrow((x \vee y) \rightarrow z)$

$$
\begin{aligned}
= & \left((x \rightarrow z)^{\prime} \vee(y \rightarrow z)^{\prime}\right)^{\prime} \rightarrow((x \vee y) \rightarrow z) \\
= & ((x \vee y) \rightarrow z)^{\prime} \rightarrow\left((x \rightarrow z)^{\prime} \vee(y \rightarrow z)^{\prime}\right) \\
= & ((x \vee y) \rightarrow z)^{\prime} \rightarrow\left(\left((x \rightarrow z)^{\prime} \rightarrow(y \rightarrow z)^{\prime}\right)\right. \\
& \left.\rightarrow(y \rightarrow z)^{\prime}\right) \\
= & ((x \vee y) \rightarrow z)^{\prime} \rightarrow(((y \rightarrow z) \rightarrow(x \rightarrow z)) \\
& \left.\rightarrow(y \rightarrow z)^{\prime}\right) \\
= & ((x \vee y) \rightarrow z) \rightarrow((x \rightarrow((y \rightarrow z) \rightarrow z)) \\
& \left.\rightarrow(y \rightarrow z)^{\prime}\right) \\
= & ((x \vee y) \rightarrow z)^{\prime} \rightarrow\left((x \rightarrow(y \vee z)) \rightarrow(y \rightarrow z)^{\prime}\right) \\
= & (x \rightarrow(y \vee z)) \rightarrow\left(((x \vee y) \rightarrow z)^{\prime} \rightarrow(y \rightarrow z)^{\prime}\right) \\
= & (x \rightarrow(y \vee z)) \rightarrow((y \rightarrow z) \rightarrow((x \vee y) \rightarrow z)) \\
= & (x \rightarrow(y \vee z)) \rightarrow((x \vee y) \rightarrow((y \rightarrow z) \rightarrow z)) \\
= & (x \rightarrow(y \vee z)) \rightarrow((x \vee y) \rightarrow(y \vee z)) \\
= & (x \vee y) \rightarrow((x \rightarrow(y \vee z))) \rightarrow(y \vee z) \\
= & (x \vee y) \rightarrow(x \vee y \vee z)=I .
\end{aligned}
$$

Hence, $(x \rightarrow z) \wedge(y \rightarrow z) \leq(x \vee y) \rightarrow z$. According to the above description (1) and (2), it can be obtained the conclusion.

Corollary 4 Let $L$ be a non-empty set, $I \in L$ be a fixed element, $\rightarrow$ be a binary operator in $L$ and satisfy $\left(L_{1}\right)-\left(L_{5}\right)$. If there exists an element $O$ in $L$, and satisfy $\forall x \in L, O \leq x$, then $\forall x, y, z \in L$,

$$
x \rightarrow(y \wedge z)=(x \rightarrow y) \wedge(x \rightarrow z) .
$$

Proof According to $\left(F_{3}\right)$ and Theorem 8, it can be obtained

$$
\begin{aligned}
x \rightarrow(y \wedge z) & =(y \wedge z)^{\prime} \rightarrow x^{\prime}=\left(y^{\prime} \vee z^{\prime}\right) \rightarrow x^{\prime} \\
& =\left(y^{\prime} \rightarrow x^{\prime}\right) \wedge\left(z^{\prime} \rightarrow x^{\prime}\right) \\
& =(x \rightarrow y) \wedge(x \rightarrow z)
\end{aligned}
$$

Theorem 9 Let $L$ be a non-empty set, $I \in L$ be a fixed element, $\rightarrow$ be a binary operator in $L$ and satisfy $\left(L_{1}\right)-\left(L_{5}\right)$. If there exists an element $O$ in $L$, and satisfy $\forall x \in L, O \leq x$, then $\forall x, y, z \in L$,

(1) $(x \wedge y) \rightarrow z=(x \rightarrow y) \rightarrow(x \rightarrow z)=(y \rightarrow$ $x) \rightarrow(y \rightarrow z)$

(2) $x \rightarrow(y \vee z)=(y \rightarrow z) \rightarrow(x \rightarrow z)=(z \rightarrow$ $y) \rightarrow(x \rightarrow y)$.

Proof 1) According to $\left(F_{3}\right)$ and Theorem 6, it can be obtained

$$
\begin{aligned}
& (x \wedge y) \rightarrow z=\left(x^{\prime} \vee y^{\prime}\right)^{\prime} \rightarrow z \\
& =z^{\prime} \rightarrow\left(\left(y^{\prime} \rightarrow x^{\prime}\right) \rightarrow x^{\prime}\right)
\end{aligned}
$$




$$
\begin{aligned}
& =\left(y^{\prime} \rightarrow x^{\prime}\right) \rightarrow\left(z^{\prime} \rightarrow x^{\prime}\right) \\
& =(x \rightarrow y) \rightarrow(x \rightarrow z) .
\end{aligned}
$$

$(x \wedge y) \rightarrow z=(y \rightarrow x) \rightarrow(y \rightarrow z)$ can be proved in the same reason.

2) According to Lemma 1 and $\left(L_{5}\right)$, it can be obtained

$$
\begin{gathered}
x \rightarrow(y \vee z)=x \rightarrow((y \rightarrow z) \rightarrow z) \\
=(y \rightarrow z) \rightarrow(x \rightarrow z)=(z \rightarrow y) \rightarrow(x \rightarrow y) .
\end{gathered}
$$

Theorem 10 Let $L$ be a non-empty set, $I \in L$ be a fixed element, $\rightarrow$ be a binary operator in $L$ and satisfy $\left(L_{1}\right)-\left(L_{5}\right)$. If there exists an element $O$ in $L$, and satisfy $\forall x \in L, O \leq x$, then $\forall x, y, z \in L$,

(1) $(x \rightarrow z) \vee(y \rightarrow z) \leq(x \wedge y) \rightarrow z$;

(2) $(x \rightarrow y) \vee(x \rightarrow z) \leq x \rightarrow(y \vee z)$.

Proof 1) According to Theorem 9, it can be obtained $(x \rightarrow z) \rightarrow((x \wedge y) \rightarrow z)=(x \rightarrow$ $z) \rightarrow((x \rightarrow y) \rightarrow(x \rightarrow z))=I$. Hence $(x \rightarrow z) \leq(x \wedge y) \rightarrow z$. As the same reason, $(y \rightarrow z) \leq(x \wedge y) \rightarrow z$ can be proved, namely $(x \rightarrow z) \vee(y \rightarrow z) \leq(x \wedge y) \rightarrow z$.

2) According to Theorem 9 ,

$$
\begin{aligned}
& (x \rightarrow y) \rightarrow(x \rightarrow(y \vee z)) \\
= & (x \rightarrow y) \rightarrow((y \rightarrow z) \rightarrow(x \rightarrow y)) \\
= & (y \rightarrow z) \rightarrow((x \rightarrow y) \rightarrow(x \rightarrow y)) \\
= & (y \rightarrow z) \rightarrow I=I .
\end{aligned}
$$

Hence, $(x \rightarrow y) \leq(x \rightarrow(y \vee z))$. As the same reason, it can be proved $(x \rightarrow z) \leq(x \rightarrow(y \vee z))$, namely $x \rightarrow(y \vee z) \geq(x \rightarrow y) \vee(x \rightarrow z)$.

Theorem 11 Let $L$ be a non-empty set, $I \in L$ be a fixed element, $\rightarrow$ be a binary operator in $L$ and satisfy $\left(L_{1}\right)-\left(L_{5}\right)$. If there exists an element $O$ in $L$, and satisfy $\forall x \in L, O \leq x$, then $\forall x, y, z \in L$, $(x \rightarrow z) \vee(y \rightarrow z)=(x \wedge y) \rightarrow z$ iff $(x \rightarrow y) \vee(x \rightarrow$ $z)=x \rightarrow(y \vee z)$.

Proof 1) If $(x \rightarrow z) \vee(y \rightarrow z)=(x \wedge y) \rightarrow z$, then

$$
\begin{aligned}
x \rightarrow(y \vee z) & =(y \vee z)^{\prime} \rightarrow x^{\prime}=\left(y^{\prime} \wedge z^{\prime}\right) \rightarrow x^{\prime} \\
& =\left(y^{\prime} \rightarrow x^{\prime}\right) \vee\left(z^{\prime} \rightarrow x^{\prime}\right) \\
& =(x \rightarrow y) \vee(x \rightarrow z)^{\prime} .
\end{aligned}
$$

2) If $(x \rightarrow y) \vee(x \rightarrow z)=x \rightarrow(y \vee z)$, then

$$
\begin{aligned}
(x \wedge y) \rightarrow z & =z^{\prime} \rightarrow(x \wedge y)^{\prime}=z^{\prime} \rightarrow\left(x^{\prime} \vee y^{\prime}\right) \\
& =\left(z^{\prime} \rightarrow x^{\prime}\right) \vee\left(z^{\prime} \rightarrow y^{\prime}\right) \\
& =(x \rightarrow z) \vee(y \rightarrow z)
\end{aligned}
$$

According to the above discussion, in $\wedge, \vee$ and ' algebra system defined by $\rightarrow$, the axioms in lattice implication algebra, except the property $(x \wedge y) \rightarrow$ $z=(x \rightarrow z) \vee(y \rightarrow z)$, satisfy the algebra system. For convenience, " $L$ be a non-empty set, $I \in L$ be a fixed element, $\rightarrow$ be a binary operator in $L^{\prime \prime}$ is marked by $(L, \rightarrow, I)$. In $(L, \rightarrow, I)$, if there exists $O$ and satisfy $\forall x \in L, O \leq x$. For $O \leq x$ iff $O \rightarrow x=I$, especially notes

$$
\left(L_{6}\right) \quad O \rightarrow x=I .
$$

Naturally, $(L, \rightarrow, I, O)$ represents that $I$ and $O$ are two fixed element, $\rightarrow$ is a binary operation in $L$.

Corollary 5 Supposed that $(L, \rightarrow, I, O)$ and $\forall x, y, z \in L, \rightarrow$ satisfy $\left(L_{1}\right)-\left(L_{6}\right)$, then $(L, \rightarrow, I, O)$ is a quasi lattice implication algebra.

Proof $\left(L_{2}\right),\left(L_{5}\right)$ and $\left(L_{3}\right)$ are respectively $\left(I_{2}\right)$, $I_{1}$ and $\left(I_{5}\right)$ in quasi lattice implication algebra [8]. According to Theorem 1 and $(2)$ of Theorem $4,\left(l_{3}\right)$ and $\left(l_{4}\right)$ in quasi lattice implication algebra can be obtained.

Theorem 12 Supposed that $(L, \rightarrow, I, O)$ and $\forall x, y, z \in L, \rightarrow$ satisfy $\left(L_{1}\right)-L_{6}$, then $(L, \vee, \wedge, I, O)$ is a distributive lattice, namely $\forall x, y, z \in L$, we have

(1) $(x \wedge y) \vee z=(x \vee z) \wedge(y \vee z)$;

(2) $(x \vee y) \wedge z=(x \wedge z) \vee(y \wedge z)$.

Proof 1) According to Lemma 1 and Corollary 4, we have

$$
\begin{aligned}
& (x \wedge y) \vee z \\
= & (z \rightarrow(x \wedge y)) \rightarrow(x \wedge y) \\
= & ((z \rightarrow x) \wedge(z \rightarrow y)) \rightarrow(x \wedge y) \\
= & (((z \rightarrow x) \wedge(z \rightarrow y)) \rightarrow x) \wedge(((z \rightarrow x) \\
& \wedge(z \rightarrow y)) \rightarrow y) \\
\geq & ((z \rightarrow x) \rightarrow x) \wedge((z \rightarrow y) \rightarrow y) \\
= & (x \vee z) \wedge(y \vee z) .
\end{aligned}
$$

It is obvious that $(x \wedge y) \vee z \leq(x \vee z) \wedge(y \vee z)$. So, $(x \wedge y) \vee z=(x \vee z) \wedge(y \vee z)$.

2) According to Theorem 6 and the conclusion (1) of Theorem 12, it can be obtained

$$
\begin{aligned}
(x \vee y) \wedge z & =\left(\left(x^{\prime} \wedge y^{\prime}\right) \vee z^{\prime}\right)^{\prime} \\
& =\left(\left(x^{\prime} \vee z^{\prime}\right) \wedge\left(y^{\prime} \vee z^{\prime}\right)\right)^{\prime} \\
& =(x \wedge z) \vee(y \wedge z)
\end{aligned}
$$


Theorem 13 Supposed that $(L, \rightarrow, I, O)$ and $\forall x, y, z \in L, \rightarrow$ satisfies $\left(L_{1}\right)-\left(L_{6}\right)$, then

$$
x \rightarrow(y \vee z)=(x \rightarrow z) \vee(x \rightarrow z)
$$

Proof According to Theorem 10, we just need to prove $x \rightarrow(y \vee z) \leq(x \rightarrow y) \vee(x \rightarrow z)$. For

$$
\begin{aligned}
& (x \rightarrow(y \vee z)) \rightarrow((x \rightarrow y) \vee(x \rightarrow z)) \\
= & (x \rightarrow(y \vee z)) \rightarrow(((x \rightarrow) \rightarrow(x \rightarrow z)) \rightarrow \\
& (x \rightarrow z)) \\
= & (x \rightarrow(y \vee z)) \rightarrow(((x \wedge y) \rightarrow z) \rightarrow(x \rightarrow z)) \\
= & (x \rightarrow(y \vee z)) \rightarrow(x \rightarrow(((x \wedge y) \rightarrow z) \rightarrow z)) \\
= & (x \rightarrow(y \vee z)) \rightarrow(x \rightarrow((x \wedge y) \vee z)) \\
= & (x \rightarrow(y \vee z)) \rightarrow((x \rightarrow(x \vee z)) \wedge(x \rightarrow \\
& (y \vee z))) \\
= & (x \rightarrow(y \vee z)) \rightarrow(I \wedge(x \rightarrow(y \vee z))) \\
= & (x \rightarrow(y \vee z)) \rightarrow(x \rightarrow(y \vee z))=I,
\end{aligned}
$$

So $x \rightarrow(y \vee z)=(x \rightarrow) \vee(x \rightarrow z)$.

Corollary 6 Supposed that $(L, \rightarrow, I, O)$ and $\forall x, y, z \in L, \rightarrow$ satisfy $\left(L_{1}\right)-\left(L_{6}\right)$, then $(L, \rightarrow, I, O)$ is a lattice implication algebra.

Proof According to Corollary 5, it can be obtained that $(L, \rightarrow, I, O)$ is quasi lattice implication algebra. According to Theorem 8 and Theorem 13, it satisfy $\left(I_{6}\right)$ and $\left(I_{7}\right)$. And according to the definition of lattice implication algebra, $(L, \rightarrow, I, O)$ is a lattice implication algebra.

\section{Conclusions}

In this paper, based on implication operation $\rightarrow$, partial order on non-empty $L$ could be induced, and generating boundary lattice on non-empty $L$ with the greatest element and the least element is discussed. Moreover, lattice implication algebra could be obtained if $\rightarrow$ satisfies $\left(L_{1}\right)-\left(L_{6}\right)$ of this paper.

\section{Acknowledgement}

This work is supported by a Project Supported by Scientific Reserch Fund of Sichuan Provincial Education Department(Grant No.2006A084), the National Natural Science Foundation of China (60474022), the Special Research Funding to Doctoral Subject of Higher Education Institutes in China (Grant No.20060613007) and the Excellent Young Foundation of Sichuan Province (Grant No.06ZQ026-037)

\section{References}

[1] Y. Xu, Lattice implication algebra. J. Southwest Jiaotong Univ, 28(1):20-27, 1993.

[2] Y. Xu, K. Qin, Lattice-valued propositional logic (I). J. Southwest Jiaotong Univ, (English version), 2:123-128, 1993.

[3] Y. Xu, K. Qin, Lattice-valued propositional logic (II), J. Southwest Jiaotong Univ, (English version), 1:22-27, 1994.

[4] Y. Xu, K. Qin, Z. Song, Syntax of lattice-valued first-order logic FM. Chin. Sci. Bull, 10:10521055, 1997.

[5] Y. Xu, K. Qin, J. Liu, Z. Song, L-valued propositional logic Lvpl. Inform. Sci, 114 :205-235, 1999.

[6] Y. Xu, J. Liu, Z. Song, K. Qin, On semantics of $L$-valued first-order logic $L v f l$. Internat. J. Gen. Systems, 29:53-79, 2000.

[7] Y. Xu, Z. Song, K. Qin, J. Liu, Syntax of $L$ valued first-order logic Lvfl. Mult.-Valued Log, 7:213-257, 2001.

[8] Y. Xu, D. Ruan, K. Qin, J. Liu, Lattice-Valued Logic-An Alternative Approach to Treat Fuzziness and Incomparability, Springer, New York, 2003. 